\title{
Distribution of Extra Hepatic Branches of Portal Vein to Caudate Lobe
}

\author{
Dr. Shilpa Gosavi ${ }^{1}$, Dr. Surekha Jadhav, ${ }^{2}$ Dr. Rajendra Garud ${ }^{3}$ \\ 1\&3 (, Department Of Anatomy, Bharati Vidyapeeth Medical College, Pune, Bharati Vidyapeeth Deemed \\ University, Pune, Maharashtra, India) \\ ${ }^{2}$ (Department Of Anatomy, PDVVPF's Medical College, Ahamednagar, Maharashtra University Of Health \\ Sciences, Maharashtra, India)
}

\begin{abstract}
The caudate lobe of the liver is anatomically distinct from left and right hepatic lobes. Kumon first divided it into three parts: Spiegel lobe, paracaval portion and caudate process. The present study was conducted to note the extra hepatic branching pattern of $P V$ and its distribution to various parts of caudate lobe and to note its variations. Eighty six cadaveric livers were studied. Number of branches from extra hepatic part of portal vein supplying different parts of caudate lobe, their origin from right branch (RPV) or left branch of portal vein (LPV) or from hilar bifurcation $(H B)$ was noted. Their distribution to the parts of caudate lobe and diameter at origin was noted. At least one separate branch to caudate lobe from extra hepatic part of PV was present in $93.02 \%$ livers. $62.11 \%$ branches were originating from LPV, $26.09 \%$ from hilar bifurcation and $11.8 \%$ were from the RPV. Spiegel lobe received $98.64 \%$ from LPV and $1.35 \%$ from HB. Paracaval portion was supplied by $43.54 \%$ branches from $L P V, 48.39 \%$ from $H B$ and $8.06 \%$ from RPV. The caudate process received $56 \%$ branches from RPV and $44 \%$ from HB. Knowledge of vascular anatomy of caudate lobe is important for hepatic surgeons and for anatomists.
\end{abstract}

Keywords: Caudate lobe, Portal vein, Spiegel lobe, Caudate process

\section{Introduction}

The caudate lobe of the liver is anatomically distinct from the left and right hepatic lobes. It is interposed between the inferior vena cava posteriorly, the left hepatic lobe anteriorly and superiorly and the portal vein inferiorly. [1]

Current understanding of the functional anatomy of the liver is based on Couinaud's division of the liver into eight (subsequently 9) functional segments, based upon the distribution of the portal venous branches and the location of the hepatic vein in the parenchyma.[2] The caudate lobe was regarded as an independent segment and was classified by Couinaud [3] as segment 1 of the liver. Kogure et al.[4] quoted that, Kumon first divided the caudate lobe into three parts: the spiegel lobe, which was equivalent to Couinaud's segment 1, the conventional caudate lobe and the paracaval portion and the caudate process.

The Spiegel lobe is located behind the lesser omentum, just to the left of intrahepatic inferior vena cava. The paracaval portion is in front of the intrahepatic IVC, just to the right of the spiegel lobe and is closely attached to the right and middle hepatic veins. The caudate process is a tongue shaped projection between the IVC and the adjacent portal vein, just to the right of the paracaval portion.[5]

Caudate lobe resection is one of the most demanding procedures among hepatic resection, owing to its deep and complex location and its proximity to major vessels. Careful technique and detailed knowledge of the caudate lobe anatomy are essential to perform isolated caudate lobectomy.[6]

The hilar bifurcation (HB) is a quadrangular area on the portal vein (PV) in which the left branch of portal vein (LPV) and the right branch of portal vein (RPV) divide from the main portal trunk. The HB roughly corresponds to an area located between two lines for ligation and incision of the left or right branch of PV during usual left or right hepatectomy without vascular reconstruction. In the human liver not only the left and right but also the HB gives off branches that supply the caudate lobe.[7]

The present study was conducted to note the extra hepatic branching pattern of PV and its distribution to the various parts of caudate lobe and to note its variations.

\section{Material and methods}

Eighty six formalin fixed cadaveric livers, without any gross abnormality or cirrhosis, were collected from Department of Anatomy of Bharati Vidyapeeth medical college, Pune and PDVVPF's medical college, Ahmednagar. Portahepatis was dissected and cleaned to identify extra hepatic branches of portal vein (PV) to the caudate lobe.

Number of branches from extra hepatic part of PV supplying different parts of caudate lobe, their origineither from right branch of portal vein (RPV), from left branch of portal vein (LPV) or from hilar 
bifurcation (HB) was noted. We also observed the diameter of these branches supplying the caudate lobe at the origin with the help of digital vernier caliper accurate up to $0.01 \mathrm{~mm}$. The distribution of these branches to the parts of caudate lobe was noted.

\section{Results}

In the present study, $93.02 \%(n=80)$ livers were showing at least one separate branch to caudate lobe from extra hepatic part of PV or its branch. The most frequently observed pattern, present in 73 livers $(84.88 \%)$ was that, the PV divided into right and the left branches. One branch arising from LPV was supplying the caudate lobe.

Number of branches from extra hepatic parts of PV supplying different parts of caudate lobe was ranging from zero to five. (Fig.1) (Table-1) Total number of branches to caudate lobe in all the livers studied was one hundred and sixty one. $62.11 \%(n=100)$ branches were originating from LPV, $26.09 \%(n=42)$ from hilar bifurcation and $11.8 \%(\mathrm{n}=19)$ were from the RPV.

Out of the 100 branches arising from LPV, 73 were supplying Spiegel lobe and 27 were for paracaval portion. 30 out of 42 branches arising from HB were supplying paracaval portion, 11 out of 42 to the caudate process and only one out of 42 branches was supplying the Speigel lobe. Out of the 19 branches arising from RPV, 14 were supplying the caudate process and five were supplying paracaval portion.

When area of distribution for these branches was considered, Spiegel lobe received 74 branches, 73 out of $74(98.64 \%)$ were from LPV and one (1.35\%) was from HB. Out of 62 branches supplying paracaval portion 27 branches (43.54\%) were arising from LPV, 30 out of 62 (48.39\%) from HB and five (8.06\%) from RPV. While the caudate process received 25 branches, 14 (56\%) were from RPV and 11 (44\%) from HB. (Table-2)

The external diameter of the branches arising from extra hepatic parts of PV was measured at its origin. Branches having diameter less than $1 \mathrm{~mm}$ were considered minor. In major branches the diameter was ranging from $1.00-3.96 \mathrm{~mm}$. The mean diameter was $1.75 \mathrm{~mm}$.

\section{Discussion}

Configurational variation of the caudate lobe subdivisions may have a significant impact on isolated caudate lobectomy for hepatocellular carcinoma restricted to Spiegel lobe.[7] Knowledge of vascular anatomy of the caudate lobe is vital in preventing and controlling hemorrhage.[8] In the present study we observed presence of separate branch for caudate lobe in $93.02 \%$ livers. Number of branches from extra hepatic part of PV to caudate lobe varied from zero to five. Kogure et al. [4] observed that the number of branches supplying the caudate lobe varied from one to six. Ortale et al. [9] observed presence of one branch to caudate lobe in $37.5 \%$, Munguti et al in $25.8 \%$, while we observed it in $18.6 \%$ cases. Ortale et al. [9] observed two or three branches in $62.5 \%$ cases, Kogure et al. [4] observed it in 32.5\%, Munguti et al. [8] observed two branches in $48.5 \%$ and three branches in $19.7 \%$, while in the present study, presence of two branches from extra hepatic part of PV or from its branches was noted in $52.32 \%$ cases and three branches were observed in $20.93 \%$ cases.Munguti et al. [8] observed presence of four branches in $6 \%$ cases while in the present study we did not observe any liver with four branches but five branches were observed in one liver. In six livers in the present study, the branch to caudate lobe from extra hepatic parts of PV was absent.

Lopez-Andújar et al. [10] observed that, the portal vein branches to the caudate lobe came predominantly from the left branch of portal vein, but it may also arise from the right branch of portal veinor the portal vein bifurcation (HB of the present study). Observations in the present study were similar. We observed that, majority of the branches $(62.11 \%)$ supplying the caudate lobe were originating from LPV, 26.09\% from HB and 11.8\% from the RPV. This observation was similar to Kogureet al. [4] and Munguti et al. [8]. Pillai et al. [6] quoted that, the ligation of large branches, about $1 \mathrm{~mm}$ thick, is a key step in caudate lobe resection. In the present study we observed $71.15 \%$ branches were more than $1 \mathrm{~mm}$ diameter at origin.

Subdivisions of the caudate lobe cannot simply be described into the right and left portions in many cases. Kanamura et al. [7] introduced the concept of HB territory and they employed this concept to determine the anatomic principal border as well as the real sub divisional configuration of the caudate lobe. They also have stated that presence or absence of HB territories has special significance when preparing the graft by living donor liver transplant.

The present study supports the observation byKanamura et al. [7] We observed presence of 43 branches in 36 livers $(41.86 \%)$ from HB supplying the caudate lobe. In $34.88 \%$ one branch was arising from HB while in $6.98 \%$ two branches were from HB. Branches from HB were supplying the paracaval portion \& caudate process in majority and the Spiegel lobe in few.

Anatomic information about the $\mathrm{HB}$ territory can provide critical information to delimit reliable margins for various types of caudate lobe surgery, such as not only routine left or right lobectomy but also extended left liver grafts with the left caudate lobe during living-related liver transplantation. The ideal 
parenchymal transaction plane for anatomic hepatectomy would differ between livers with or without HB territory. [7]

In the present study caudate process was supplied by branches from RPV (56\%) mainly and also from HB (44\%) but not from LPV. Ortale et al. [9] observed 38.09\% branches were arising from RPV while Kogure et al. [4] observed 50.5\% from RPV but they also noted few branches from LPV. The paracaval portion in the present study was supplied by branches from HB in $48.38 \%$, LPV in $43.54 \%$ and RPV in $8.06 \%$ cases. These findings were similar to Ortale et al. [9] (HB- 50\%, LPV- 21.48\% and RPV - 2.85\%) but not with Kogure et al. [4] ( $\mathrm{HB}-38.51 \%, \mathrm{LPV}-24.84 \%$ and RPV $-36.64 \%)$.

Knowledge of vascular anatomy of caudate lobe is important for hepatic surgeons for isolated caudate lobectomy or for preventing and controlling hemorrhage during various procedures. It is also important for anatomists.

\section{Figures and Tables}

Table 1: Table showing number of branches supplying caudate lobe

\begin{tabular}{|c|c|}
\hline No. of branches & $\begin{array}{c}\text { Present in (\%) } \\
\mathrm{n}=86\end{array}$ \\
\hline 0 & $06(6.98)$ \\
\hline 1 & $16(18.6)$ \\
\hline 2 & $45(52.32)$ \\
\hline 3 & $18(20.93)$ \\
\hline 4 & 00 \\
\hline 5 & $01(1.16)$ \\
\hline
\end{tabular}

Table 2: Distribution of branches to different parts of caudate lobe

\begin{tabular}{|c|c|c|c|}
\hline & Spiegel lobe $(\mathrm{n}=74)$ & Paracaval portion $(\mathrm{n}=62)$ & Caudate process $(\mathrm{n}=25)$ \\
\hline LPV & $73(98.64 \%)$ & $27(43.54 \%)$ & 00 \\
\hline HB & $01(1.35 \%)$ & $30(48.38 \%)$ & $11(44 \%)$ \\
\hline RPV & 00 & $05(8.06 \%)$ & $14(56 \%)$ \\
\hline
\end{tabular}

(LPV - left branch of portal vein, HB - Hilar bifurcation, RPV - right branch of portal vein)

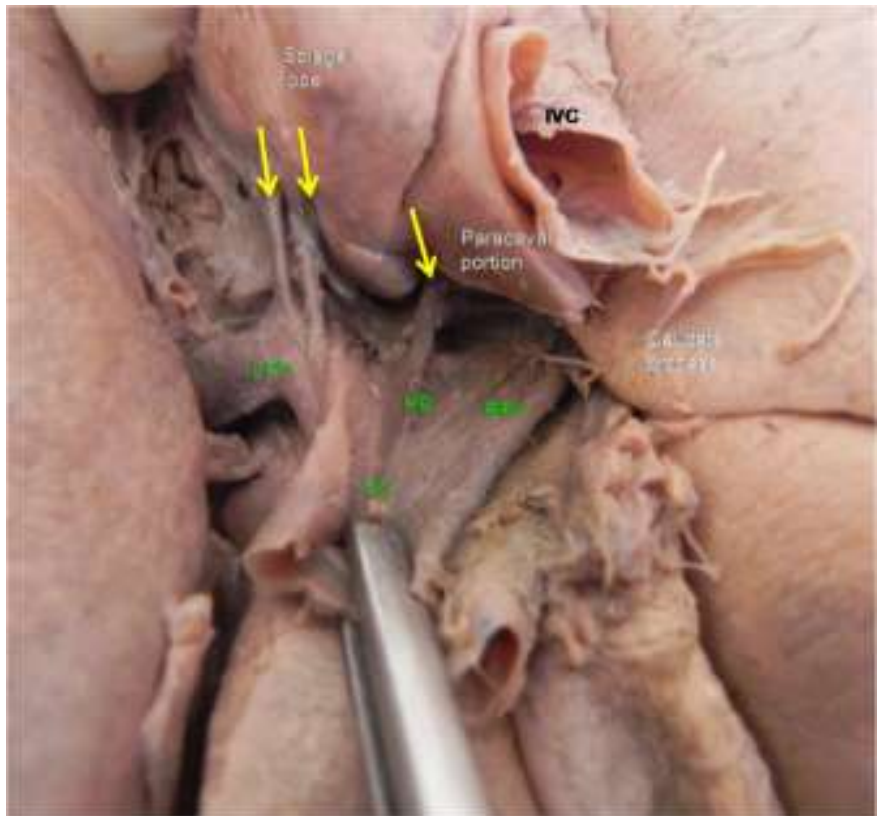

Fig1- Photograph showing divisions of caudate lobe and branches of portal vein supplying it (HB - Hilar bifurcation, LPV - Left branch of portal vein, RPV - Right branch of portal vein Yellow arrows showing extra hepatic branches of portal vein to caudate lobe)

\section{Conclusion}

In the cadaveric study of extra hepatic branches supplying the caudate lobe in eighty six livers, we observed maximum number of branches to caudate lobe were from LPV. Spiegel lobe was mainly getting branches from LPV, paracaval portion mainly from HB and caudate process from RPV and also from HB. Majority of the branches to caudate lobe had external diameter $>1 \mathrm{~mm}$ at origin. Knowledge of vascular anatomy 
of caudate lobe is important for hepatic surgeons for isolated caudate lobectomy or for preventing and controlling hemorrhage during various procedures. It is also important for anatomists.

\section{References}

[1]. Brown BM, Filly RA, Callen PW. Ultrasonographic anatomy of the caudate lobe. J Ultrasound Med; 1, 1982, $189-192$.

[2]. Standring S. (Ed). Gray's Anatomy: the Anatomical basis of clinical practice(40 ${ }^{\text {th }}$ edn. London, UK:Elsvier ltd, 2008) 1165.

[3]. CouinadeC.Portal segmentation. In: Couinad C, (Ed). Controlled hepatectomies and exposure of the intrahepatic bile ducts(Paris: 1981) 9-27.

[4]. Kogure K, Kuwano H, Fujikama N, Makuuchi M. Relation among portal segmentation, proper hepatic vein and external notch of the caudate lobe in the human liver. Annals of surgery 231(2),2000, 223-228.

[5]. Wen ZQ, Yan YQ, Yang JM, Wu MC.Precautions in caudate lobe resection: Report of 11 cases. World J Gastroenterol 14(17),2008, 2767-2770.

[6]. Pillai SA, Sathyanesan J, Perumal S, Perumal SU, Lakshman A, Ramswami S, Ramswami R. Isolated caudate lobe resection: technical challenges. Ann Gastroenterol 26(1),2013, 1-6.

[7]. Kanamura T, Murakami G, Ko S, Hirai I, Hata F, Nakajima Y. Evaluating the Hilar bifurcation territory in the human liver caudate lobe to obtain critical information for delimiting reliable margins during caudate lobe surgery: anatomic study of livers with and without the external caudate notch. World J. Surg.; 27,2003, 284-288.

[8]. Munguti J, Sammy M, Odula P, awori K.Portal venous branches to the caudate lobe: number and origin. Anatomy journal of Africa 3(1),2014, 228-231.

[9]. Ortale JR, Borges Keiralla LC. Anatomy of the portal branches and the hepatic veins in the caudate lobe of the liver. SugrRadiolAnat 26, 2004, 384-391.

[10]. Lopez-Andújar R, Montalva E, Pareja E, Mir J. Step-by-step isolated resection of segment 1 of the liver using the hanging maneuver. Am J Surg 198, 2009, e42-e48. 